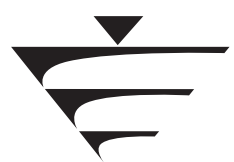

\title{
Addendum: Use of risk-based methods to evaluate spillway capacity: case histories
}

Alan Brown MA, MSC, DIC, CEng, FICE, MCIWEM

All Reservoirs Panel Engineer, Technical Director, Stillwater Associates, Redhill, Surrey, UK

John Gosden MA, MSC CEng, FICE, MCIWEM

All Reservoirs Panel Engineer, Senior Consultant, Jacobs, Winnersh, Wokingham, Berkshire, UK

Martin Hewitt BSC, CEng, FICE

All Reservoirs Panel Engineer, Technical Director, Mott MacDonald, Glasgow, UK
Jonathan Hinks MA, MSc, CEng, FICE

All Reservoirs Panel Engineer, Technical Director, HR Wallingford, Wallingford, UK

Keith Gardiner BSC, CEng, FICE

All Reservoirs Panel Engineer, Atkins, Warrington, UK
The authors regret that the following error appeared in this paper when it was published in Dams and Reservoirs 24(3): 120-133.
The affiliation for Keith Gardiner was listed incorrectly. It should have been as above. 